cours de la première phase, l'action de l'alcool isoamylique provoque une accélération des battements du cœur, il s'agit de la phase d'excitation. Au cours de la seconde phase, un ralentissement du rythme respiratoire est observé.

L'alcool isoamylique détruit les émulsions de la matière grasse par abaissement de la tension superficielle, tel est son rôle dans les analyses de lait et de crème. N'est-il pas permis alors de se demander s'il n'aurait pas une action du même ordre sur le système nerveux ? Evidemment, seules des recherches plus approfondies pourraient permettre d'élucider l'action de cet alcool sur l'organisme.

Il serait très intéressant de connaître la concentration limite dans l'atmosphère pouvant être supportée sans inconvénient. Aussi il nous paraît indispensable que les conditions dans lesquelles l'alcool isoamylique peut être employé dans les laboratoires de laiteries, fassent l'objet d'une étude sérieuse et que cette étude soit suivie d'une règlementation si cela s'avère nécessaire, comme nos observations le laissent supposer.

Si nous avons cru utile de relater ces observations, malheureusement trop sommaires, c'est parce qu'il nous a semblé opportun de poser un problème dont la solution intéresse la santé de tout le personnel employé dans les laboratoires d'analyses des laiteries.

\title{
APPAREIL A PROJECTION POUR LA LECTURE PRÉCISE DES BUTYROMETRES (1)
}

\author{
par \\ JEAN PIEN
}

L'une des conditions primordiales de l'exactitude des résultats obtenus dans le dosage de la matière grasse du lait par la méthode Gerber est la précision de la lecture du nombre de divisions occupé par la colonne grasse.

Or de nombreuses causes d'erreur peuvent intervenir à ce stade :

1) Déplacement spontané de la colonne grasse: dès que le butyromètre est sorti du bain-marie à $65^{\circ}$, le liquide hydro-acide contenu dans la panse se refroidit, se contracte, et le niveau occupé par la colonne grasse tend à descendre. En se déplaçant, la colonne grasse tapisse l'intérieur du tube gradué d'un film gras qui ne se rassemble pas immédiatement dans la couche grasse principale et diminue d'autant la longueur apparente de cette colonne.

(1) Communication au XIVe Congrès International de Laiterie (Rome, septembre 1956). 
2) Déplacement provoqué de la colonne grasse: pour remédier à l'inconvénient précédent, l'opérateur agit sur le bouchon et déplace la colonne grasse dans la tige graduée. En outre, pour parvenir à placer le plan de séparation des deux phases sur une graduation principale de l'échelle, l'opérateur fait monter ou descendre l'ensemble de la colonne par une manouvre appropriée du bouchon. Cette opération a également pour résultat de tapisser l'intérieur du tube d'un film gras et, accentuant l'inconvénient précédent, la longueur de la colonne grasse se trouve diminuée.

3) Projection de matière grasse dans l'ampoule terminale: l'opération de réglage dont il vient d'être parlé exige un entraînement et une certaine habileté de la part de l'opérateur. Si ces conditions ne sont pas réalisées, il peut arriver, à la suite d'une poussée trop brusque sur le bouchon (ou d'une détente un peu brutale du bouchon trop serré) que de la matière grasse soit projetée vers l'ampoule du butyromètre. On remédie à cet accident en replaçant le butyromètre quelques instants dans le bain-marie; mais il peut arriver qu'il passe inaperçu et le résultat se trouve alors entaché d'une grave erreur.

4) Contraction de la colonne grasse: si la lecture n'est pas extrêmement rapide (quelques secondes) la colonne grasse se refroidit, se contracte et présente un volume inférieur à ce qu'il aurait dû être.

5) Déplacement de la colonne grasse pendant la lecture: lorsque l'opérateur a mis le plan inférieur de la colonne grasse en coïncidence avec une division principale de l'échelle graduée, il déplace le butyromètre devant l'œil pour lire le niveau du point le plus bas du ménisque supérieur. Pendant cette opération, quoique rapide, le plan inférieur a pu se déplacer légèrement puisque, du fait du refroidissement du contenu de la panse, la colonne grasse tend toujours à descendre. La pression continue opérée sur le bouchon pour s'opposer à cette descente a pu, au contraire, faire remonter légèrement la colonne grasse. Si ce déplacement est passé inaperçu, la lecture est faussée. L'opérateur doit donc (comme il est prescrit dans la méthode officielle française) [1] «vérifier immédiatement le plan de séparation inférieur de la colonne grasse pour s'assurer qu'il n'a pas bougé ; s'il s'est déplacé, corriger la position par une manœuvre appropriée du bouchon et relire immédiatement la position du ménisque supérieur. Si le plan inférieur ne s'est pas déplacé, cette deuxième visée doit donner la même valeur que lors de la première lecture. Si la deuxième valeur est différente de la première, vérifier une fois de plus la position du plan horizontal inférieur et procéder à une troisième lecture. Il faut absolument aboutir à. ce résultat : deux lectures consécutives du ménisque supé- 
rieur doivent donner la même valeur. C'est la preuve de la constance de position du plan horizontal inférieur ".

Cette opération est donc loin d'être simple et c'est là une cause d'erreur fréquente, surtout chez les opérateurs non entraînés. Et si, pour parvenir à un résultat qu'il croit plus précis, l'opérateur s'attarde plus de quelques secondes à ces lectures successives, l'une des causes d'erreur signalées précédemment (contraction de la colonne grasse) intervient à son tour.

6) Erreurs de parallaxe: la lecture, pour être correcte, doit se faire par des visées parfaitement horizontales. Le butyromètre doit être maintenu verticalement et être déplacé devant l'œil de telle sorte que chacune des deux extrêmités de la colonne grasse soit amenée successivement dans le plan horizontal qui passe par l'œil de l'opérateur. Trop souvent, le butyromètre est maintenv. immobile et l'une des deux visées, au moins, est oblique. Il en résulte une erreur de parallaxe qui, faussant l'appréciation de la position du ménisque supérieur, conduit à des résultats trop faibles.

7) Manque de précision de la lecture: la lecture à l'œil nu permet d'apprécier approximativement la demi-division de l'échelle graduée, mais n'autorise pas une lecture plus précise. On estime, en général, qu'une précision plus grande serait inutile "puisque la méthode Gerber ne permet pas une exactitude supérieure au demigramme par litre ou par kilo de lait». Nous pensons au contraire que la méthode Gerber, appliquée correctement avec des butyromètres parfaitement calibrés et des prises d'essai rigoureuses, permet d'atteindre une exactitude supérieure à ce demi-gramme, si les conditions de la lecture peuvent également être améliorées [2].

$$
*^{*} *
$$

Ces nombreuses causes d'erreur ou d'imprécision ont conduit divers auteurs à tenter d'améliorer les conditions de la lecture des butyromètres.

La Maison Gerber a créé un modèle de loupe qui facilite l'appréciation de la position des extrémités de la colonne grasse, mais ne supprime pas la plupart des inconvénients précédents.

Posthumus a décrit un dispositif [3] qui améliore considérablement les conditions de la lecture de la colonne grasse. Dans cet appareil, les butyromètres sont disposés dans un bain-marie à paroi vitrée au travers de laquelle la lecture peut se faire au moyen d'une loupe. Tous les inconvénients dus au refroidissement se trouvent done éliminés. D'autre part, un dispositif mécanique simple permet de comprimer les bouchons de caoutchouc sans avoir à sortir les 
butyromètres du bain et facilite ainsi la mise en coïncidence du plan inférieur de la colonne grasse avec une division de l'échelle.

Cet appareil, en dépit de l'amélioration très sensible qu'il apporte, ne résout cependant pas toutes les difficultés et ne supprime pas toutes les causes d'erreur énumérées plus haut : le déplacement de la colonne grasse (si faible soit-il), résultant de la manœuvre mécanique du bouchon tapisse de matière grasse une petite portion intérieure du tube gradué; l'erreur de parallaxe (dont le danger est accru par l'emploi d'une loupe) n'est pas éliminée si l'œil ne se place pas exactement au foyer de la lentille dans les deux positions de lecture et si l'axe optique de cette lentille n'est pas amené successivement en coïncidence avec les deux extrémités de la colonne grasse (pour éviter ces erreurs de parallaxe les yeux de l'opérateur doivent être appliqués et pressés, dans un cadre en fil métallique solidaire de la loupe); et le bain-marie, qui n'est pas thermostatique, est réglé à $60^{\circ}$ et non à $65^{\circ}$ pour obtenir une meilleure concordance avec les "lectures manuelles " (il y a là une objection, car les butyromètres sont gradués pour être lus à $\left.65^{\circ}\right)$; une cause d'erreur, ou au moins de difficulté de lecture, réside dans le fait que ce dispositif ne permet pas d'amener le plan inférieur de la colonne devant une division principale de l'échelle, mais seulement devant une division quelconque, de sorte que le décompte du nombre total de graduations nécessite une opération mentale qui, dans le travail en grande série, peut engendrer des erreurs; enfin et surtout, la précision de la lecture n'est pas notablement accrue car la position exacte du sommet du ménisque entre deux graduations successives ne peut pas être appréciée avec plus de certitude que dans le cas de la loupe Gerber.

Signalons encore que, dans cet appareil, l'orientation convenable des butyromètres (pour que le plan de l'échelle graduée soit perpendiculaire à l'axe optique) n'est pas automatique mais doit être assurée manuellement lors de l'immersion des butyromètres et, enfin, que les butyromètres doivent être préalablement sélectionnés, car ceux dont l'axe du tube gradué n'est pas exactement dans le prolongement de l'axe de la painse se brisent sous l'influence de la forte pression à laquelle ils sont soumis dans l'appareil.

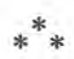

De notre côté, nous avons réalisé un appareil de lecture qui nous semble échapper à toute les critiques précédentes et qui permet l'évaluation très précise, sans erreur possible, du nombre de graduations occupé par la colonne grasse. La conception de 
cet appareil est antérieure à la parution de l'étude de Posthumus et les améliorations successives que nous lui avons fait subir en ont retardé la publication jusqu'à maintenant.

Le principe du dispositif que nous présentons est le suivant :

Le butyromètre est suspendu dans un bain-marie électrique thermostatique pourvu de deux fenêtres en matière plastique. Une lanterne électrique munie d'un condensateur éclaire vivement le butyromètre. Les rayons lumineux, qui ont traversé le bainmarie, pénètrent dans un objectif à projection et l'image ainsi formée est reçue sur un miroir de surface à $45^{\circ}$ puis renvoyée sur la plaque dépolie horizontale d'une grande chambre noire où se forme l'image réelle, agrandie trois fois, de l'échelle graduée du butyromètre et de la colonne grasse. Sur cette plaque dépolie (mobile dans le plan horizontal) est gravée une ligne de foi que l'on amènera en coïncidence avec le plan inférieur de la colonne grasse. Sur la plaque dépolie elle-même se déplace un réticule en matière plastique portant une seconde ligne de foi (Voir Fig. 1, la vue d'ensemble de l'appareil) (1).

La lecture s'effectue de la manière suivante: l'image du butyromètre étant obtenue sur la plaque dépolie (la mise au point ayant été faite une fois pour toutes) faire glisser la plaque dépolie pour amener sa ligne de foi en coüncidence avec le plan inférieur de la colonne grasse; puis, faire glisser le réticule pour amener sa ligne de foi au point de tangence avec le sommet du ménisque de la colonne grasse; celle-ci se trouve done très exactement limitée par les deux lignes de foi (voir fig. 2). Ramener, en déplaçant la plaque dépolie, la ligne de foi inférieure en coïncidence avec le zéro de l'échelle graduée du butyromètre; la ligne de foi supérieure donne alors, avec beaucoup de précision, le nombre exact de graduations (et la fraction de graduation) occupé par la colonne grasse (voir fig. 3). Ces trois opérations successives ne demandent que quelques secondes. La lecture ainsi effectuée permet d'apprécier le $1 / 5^{\mathrm{e}}$ ou même le $1 / 10^{\mathrm{e}}$ de graduation.

Remarque. - Le support des butyromètres dans le bain-marie est constitué par un carrousel à sept places où les butyromètres (prélevés d'un bain-marie ordinaire où on les a placés au sortir de la centrifugeuse) achèvent d'équilibrer leur température avant de passer devant le faisceau lumineux. Le mode de suspension des butyromètres dans le carrousel assure automatiquement leur orientation correcte. Au moment où l'on extrait le butyromètre qui vient d'être lu, le carrousel est déplacé d'un septième de tour pour amener en position le butyromètre suivant et, en même temps,

(1) Constructeur : Etablissements Beaudoin, 1, rue Rataud, Paris (5e). 


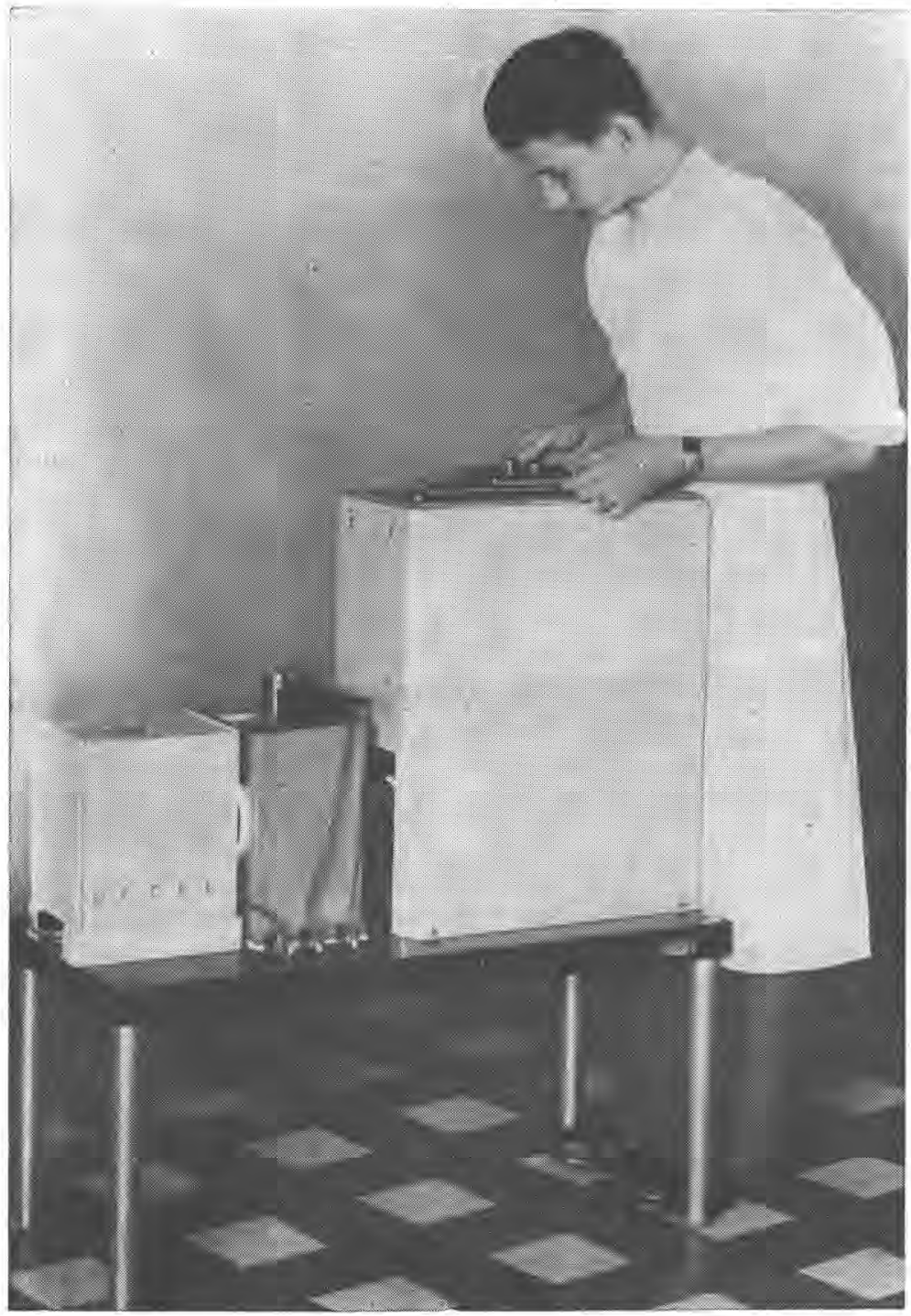

FIG. 1 


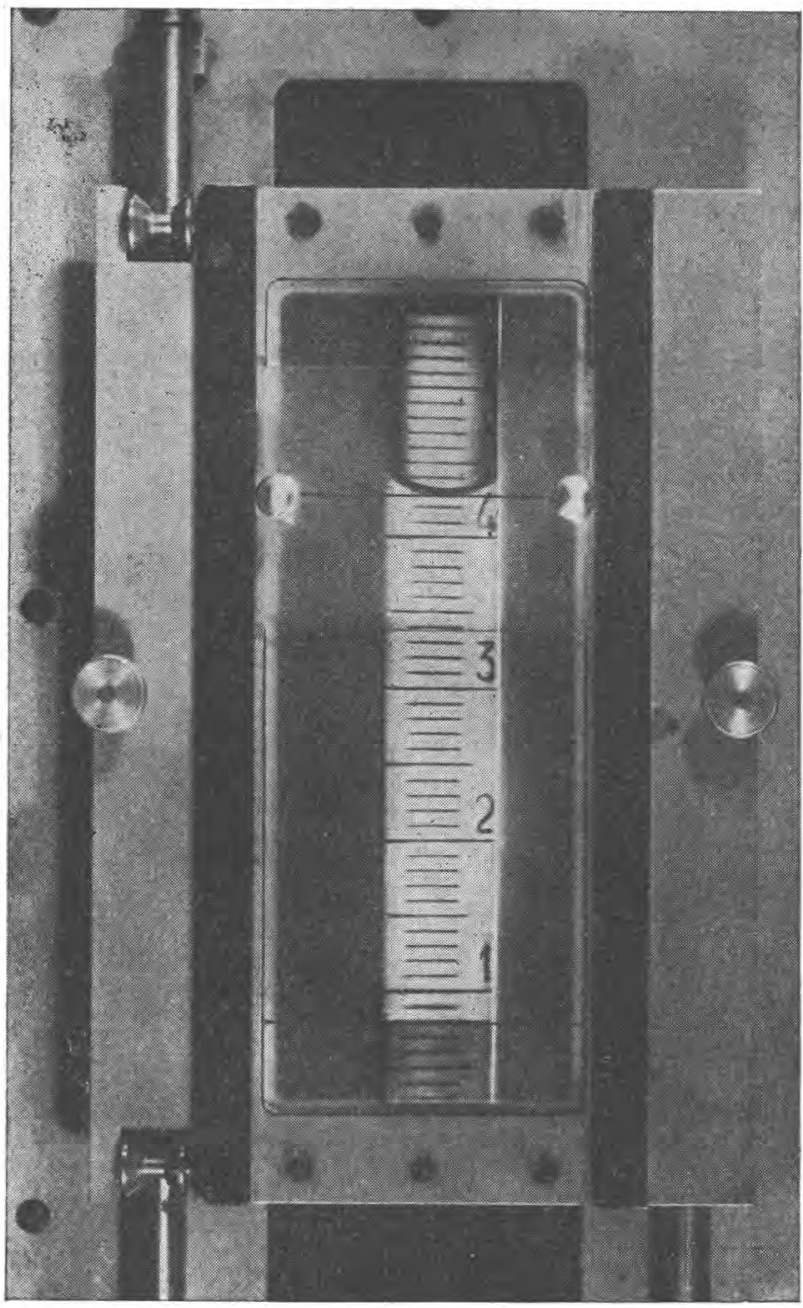

FIG. 2 


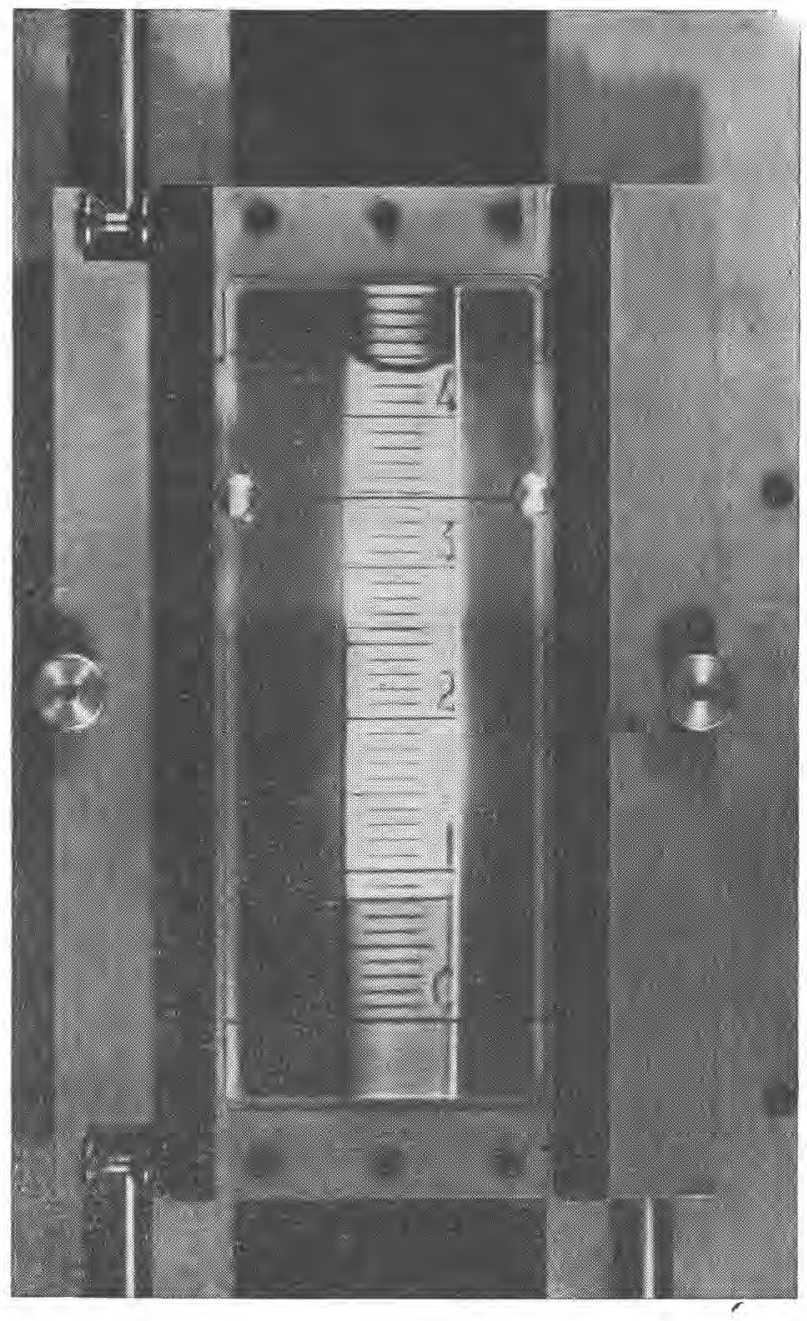

FIG. 3 
un nouveau butyromètre est introduit à l'entrée du carrousel. Cette manière de faire assure un équilibre thermique très précis et permet des manipulations aisées et rapides.

\section{Résultats obtenus}

1) L'image d'une petite division de l'échelle graduée occupe, sur l'écran, une longueur de 3 à $4 \mathrm{~mm}$., suivant les butyromètres. Par l'usage de la ligne tracée sur le réticule mobile, on peut apprécier le $1 / 10^{\mathrm{e}}$ de division.

2) Plusieurs lectures successives d'un même butyromètre (avec remise en place des deux lignes de foi) sont absolument concordantes dans la majorité des cas. Dans quelques cas, on constate un écart de $1 / 10^{\mathrm{e}}$ de division ( 0 gr. 1 par litre ou par kilo) qui correspond à l'incertitude opératoire ou à l'incertitude de lecture $( \pm 0,05$ division).

3) En analysant un même lait avec 10 butyromètres courants pris au hasard et en faisant trois lectures de chaque butyromètre, la totalité des résultats montre une dispersion maximum de \pm 0 gr. 15 par litre ou par kilo, autour de la moyenne. Une partie de cette dispersion provient des incertitudes signalées ci-dessus. Le reste ( \pm 0 gr. 1 par litre ou par kilo) provient des butyromètres eux-mêmes.

4) En comparant, sur de nombreux laits différents, les lectures faites à l'œil nu par la méthode habituelle et les lectures faites dans notre appareil (les deux lectures étant faites sur chaque butyromètre) on constate les écarts suivants :

Dans $50 \%$ des cas, la lecture à l'œil nu est déficitaire de 0,1 à 0 gr. 3 par litre de lait.

Dans $25 \%$ des cas, la lecture à l'œil nu est excédentaire de 0,1 à 0 gr. 2 par litre de lait.

Dans $25 \%$ des cas il y a identité (écart inférieur à 0 gr. 1 par litre)

5) En améliorant les conditions de la prise d'essai à l'aide de pipettes correctes [4] et en travaillant avec des butyromètres de précision (dont le tube gradué est en verre calibré de section constante et dont les graduations sont rigoureusement équidistantes) les résultats sont encore meilleurs et la précision atteint facilement \pm 0 gr. 1 par litre ou par kilogramme - ce qui permet de dire que, ainsi améliorée, la méthode Gerber devient parfaitement comparable aux méthodes pondérales.

\section{BIBLIOGRAPHIE}

[1] Méthode officielle française pour le dosage de la matière grasse du lait par la méthode acido-butyrométrique de Gerber. Arrêté Ministériel 
du 31 mars 1954 (J.O. 23 avril 1954) et Norme française. AFNORV 04-214, homologuée le 31 mars 1954 (J.O. 23 avril 1954).

[2] J. Pien. L'avenir de la méthode Gerber. C.R. du Congrès Section III. Question 4.

[3] G. Posthumus. Netherl. Milk and Dairy Journ, V. (1951), 10.

[4] J. Pien. Conditions à remplir par les pipettes à lait pour butyrométrie. C. R. du Congrès, Section III. Question 4.

\title{
LE LAIT DANS LE MONDE
}

\author{
PAR \\ G. GENIN \\ Ingénieur E. P. C.
}

\section{ANGLETERRE}

\section{La stérilisation à très haute température du lait}

$\mathrm{Au}$ Congrès de la Society of Dairy Technology, qui s'est tenu à Bournville, le Dr L.F.L. ClegG du National Institue for Research in Dairying a présenté une communication sur les tendances futures dans l'évolution des procédés de stérilisation du lait.

Une de ces tendances se manifeste par l'adoption de températures de stérilisation de plus en plus élevées, la durée du traitement étant par contre sensiblement réduite. Les raisons de cette tendance sont dues au fait que certains microorganismes, qui sont la cause de l'altération du lait pasteurisé ou du lait stérilisé, ne sont pas détruits par les méthodes actuelles de traitement.

On oublie en effet trop souvent de se rappeler que le lait stérilisé n'est pas en général rigoureusement stérile et qu'il peut donc s'altérer s'il est conservé dans des conditions défectueuses, surtout en été. Ce n'est que par un traitement à plus haute température qu'on parvient à détruire ces germes, mais un tel traitement a pour conséquence généralement d'altérer le goût du lait. On recherche done des procédés industriels permettant d'atteindre ces hautes températures, se façon à détruire les spores des bactéries, la durée de traitement du lait ne dépassant pas 1 à 2 secondes.

\section{Nouveau dispositif de distribution du lait}

Un nouveau système de distribution du lait a été récemment utilisé avec succès au milk-bar du National Milk Publicity Council, faisant partie de l'Espoxition britannique intitulée "La Maison idéale ". Pour distribuer le lait à 10 points de vente différents du Bar, il avait été prévu deux réservoirs cylindriques horizontaux, d'une capacité de près de 500 litres, exécutés en acier stainless et recevant de l'air comprimé sous une pression d'environ 200 grammes 